\title{
Skeletal effects of nongenomic thyroid hormone receptor beta signaling
}

\author{
Richard C Lindsey $1,2,3$, Catrina Godwin ${ }^{1}$ and Subburaman Mohan ${ }^{1,2,3,4,5}$ \\ ${ }^{1}$ Musculoskeletal Disease Center, VA Loma Linda Healthcare System, Loma Linda, California, USA \\ ${ }^{2}$ Center for Health Disparities and Molecular Medicine, School of Medicine, Loma Linda University, Loma Linda, California, USA \\ 3Division of Biochemistry, Department of Basic Sciences, School of Medicine, Loma Linda University, Loma Linda, California, USA \\ ${ }^{4}$ Department of Medicine, School of Medicine, Loma Linda University, Loma Linda, California, USA \\ 5Department of Orthopedics, School of Medicine, Loma Linda University, Loma Linda, California, USA
}

Correspondence should be addressed to S Mohan: subburaman.mohan@va.gov

\begin{abstract}
Thyroid hormone (TH) levels increase rapidly during the prepubertal growth period in mice, and this change is necessary for endochondral ossification of the epiphyses. This effect of TH on epiphyseal chondrocyte hypertrophy is mediated via TR $\beta 1$. In addition to its traditional genomic signaling role as a transcription factor, TR $\beta 1$ can also exert nongenomic effects by interacting with other signaling molecules such as PI3K. To investigate the role of nongenomic TR $\beta 1$ signaling in endochondral ossification, we evaluated the skeletal phenotype of TR $\beta 147 \mathrm{~F}$ mutant mice which exhibit a normal genomic response of TR $\beta 1$ to TH, but the nongenomic response through the PI3K pathway is impaired. Using microCT, we found that 13-week-old TR $\beta 147 \mathrm{~F}$ mice had significantly less trabecular bone mass at three sites. Histomorphometric analyses revealed that mineralizing surface to bone surface and BFR/BS were reduced in the mutant mice. Mechanistically, we found that activation of TR $\beta$ increased Alp and OsX expression in control but not TR $\beta 147 \mathrm{~F}$ osteoblasts. Since canonical $\beta$-catenin signaling has been implicated in mediating nongenomic TR $\beta$-PI3K signaling, we evaluated the effect of TR $\beta 1$ activation on $\beta$-catenin target gene expression in MC3T3-E1 preosteoblasts. We found that $\beta$-catenin target genes were increased, suggesting that nongenomic TR $\beta 1$-PI3K pathway modulation of $\beta$-catenin signaling may mediate TR $\beta 1$ effects on osteoblast differentiation. Together, these results suggest that $\mathrm{TH}$ acting through TR $\beta 1$ regulates endochondral ossification in part via nongenomic signaling in mice. Further investigation of this nongenomic mechanism of TR $\beta 1$ signaling could lead to novel therapeutic targets for treatment and prevention of osteoporosis.
\end{abstract}

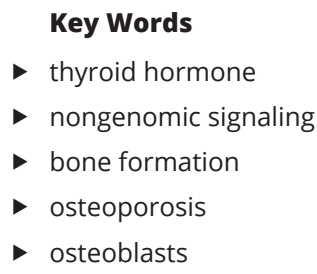

Journal of Endocrinology (2019) 242, 173-183

\section{Introduction}

Osteoporosis, a debilitating condition in which an increase in bone resorption is not adequately compensated by a corresponding increase in bone formation, leading to net loss of bone mass and microarchitectural deterioration, affects millions worldwide (Drake \& Khosla 2019). Despite the existence of current therapies, the economic and health burden of osteoporosis is expected to rise, suggesting an urgent need for a more complete understanding of the molecular pathways involved in bone formation in order to develop more effective therapeutic strategies. A key regulator of skeletal development and bone mass 
accretion is TH (Gouveia et al. 2018, Lindsey et al. 2018, Williams \& Bassett 2018). Our previous studies have shown that there is a crucial prepubertal growth period in mice during which serum thyroid levels rise; during this period, TH has a greater effect on skeletal development than growth hormone and is required for the epiphyseal ossification which occurs at that stage (Xing et al. 2012, 2014).

$\mathrm{TH}$ exerts its effects by binding to the $\mathrm{TH}$ receptors (TRs), of which there are two types: TR $\alpha$ and TR $\beta$. TR $\alpha 1$ and TR $\beta 1$ are expressed in many tissue types, but their relative expression levels and effects vary by developmental stage and tissue type. Thus, TH has many effects throughout the body, and the relative abundance of TR $\alpha$ and TR $\beta$ contribute to the specific effects TH will have in a given context (Forrest et al. 1990). The primary mechanism by which the TRs mediate the effects of $\mathrm{TH}$ is by acting as nuclear receptors, ligand-inducible transcription factors which regulate the expression of target genes. However, recent studies have determined that $\mathrm{TH}$ can exert nongenomic effects in addition to its canonical role in regulating gene expression. A number of mechanisms for these nongenomic effects have been identified. For example, several truncated TR $\alpha$ isoforms which are not transcriptionally competent can bind $\mathrm{TH}$ and regulate cellular functions by modifying targets such as mitochondrial respiration and the state of cellular actin. Furthermore, TH can bind to and modulate the activity of cytoplasmic proteins including ketamine reductase and pyruvate kinase. Additionally, TH can bind directly to the $\alpha \mathrm{V} \beta 3$ integrin to regulate proliferation and survival in cancer cells (Davis et al. 2018). Notably, TH has been shown to regulate PI3 kinase (PI3K) activity by binding to TR $\beta$ in a cytoplasmic complex with the p85 subunit of PI3K and Src family tyrosine kinase Lyn (Martin et al. 2014).

In recent studies, we have found that $\mathrm{TH}$ effects on epiphyseal bone formation are mediated primarily via TR $\beta 1$ induction of Indian hedgehog/osterix signaling in chondrocytes to promote their transdifferentiation into bone matrix-producing osteoblasts (Xing et al. 2016, Aghajanian et al. 2017). To address the question of whether TR $\beta 1$ effects on bone formation are mediated via genomic and/or nongenomic signaling pathways, we used a mouse model developed in Dr David Armstrong's laboratory with intact genomic but disrupted nongenomic signaling (Martin et al. 2014). These mutant mice have a single amino acid substitution in TR $\beta 1$ : tyrosine 147 is replaced by a phenylalanine (TR $\beta 147 \mathrm{~F}$ mice). The mutant
TR $\beta 1$ in these TR $\beta 147 \mathrm{~F}$ mice binds DNA to regulate gene expression as usual, but its nongenomic TR $\beta-\mathrm{PI} 3 \mathrm{~K}$ signaling is disrupted. The mutant TR $\beta 1$ is able to bind PI3K subunit p85, but it does not bind Lyn, and the ability of TH to regulate PI3K signaling is thus abolished (Martin et al. 2014). We evaluated the skeletal phenotype of the TR $\beta 147 \mathrm{~F}$ mice and began investigating which downstream pathway might be mediating the nongenomic TR $\beta$-PI3K effect on the skeleton.

\section{Materials and methods}

\section{Mice}

TR $\beta 147 \mathrm{~F}$ mice with intact genomic TR $\beta$ signaling but disrupted nongenomic TR $\beta$-PI3K signaling were obtained from Dr David Armstrong (Laboratory of Neurobiology, National Institute of Environmental Health and Sciences, National Institutes of Health/Department of Health and Human Services) (Martin et al. 2014). Heterozygous littermate controls were used as controls to enable us to use a breeding scheme (heterozygous $\times$ mutant) which would allow for the generation of a higher number of mutant mice. Mice were group housed at the Veterinary Medical Unit of the VA Loma Linda Healthcare System (Loma Linda, CA, USA) under standard approved, pathogen-free laboratory conditions and fed ad libitum with Teklad $4 \%$ Fat Mouse/Rat Diet (7001, Harlan Laboratories, Madison, WI, USA). This study was approved by the Institutional Animal Care and Use Committee of the VA Loma Linda Healthcare System (Loma Linda).

\section{Cell culture}

Primary calvarial osteoblasts were obtained from newborn TR $\beta 147 \mathrm{~F}$ mice and their heterozygous littermate controls as previously described (Miyakoshi et al. 2001). Briefly, calvariae were isolated via enzymatic digestion with collagenase I $(2 \mathrm{mg} / \mathrm{mL})$ and hyaluronidase $(1 \mathrm{mg} / \mathrm{mL})$. MC3T3-E1 pre-osteoblasts were obtained from ATCC. Cells were maintained in DMEM growth medium supplemented with $10 \%$ fetal bovine serum, penicillin (100units $/ \mathrm{mL}$ ) and streptomycin $(100 \mu \mathrm{g} / \mathrm{mL})$. Osteoblastic cells were grown to confluence, synchronized by serum starvation and treated with TR $\beta$-specific agonist GC-1 $(0.1 \mathrm{nM}$; Tocris Bioscience) or TR $\beta$-specific agonist MGL-3196 $(1 \mu \mathrm{M}$, Madrigal Pharmaceuticals, West Conshohocken, PA, USA) for 3 days before RNA extraction. 


\section{Micro-computed tomography}

The trabecular bone phenotype of 13-week-old TR $\beta 147 \mathrm{~F}$ mice and their control littermates of both genders was evaluated at the femoral secondary spongiosa, tibial epiphysis and lumbar vertebra L5 via micro-computed tomography (microCT; Scanco vivaCT 40, Scanco Medical, Bruttisellen, Switzerland). The bones were scanned at a resolution of $10.5 \mu \mathrm{m}$ with a $55 \mathrm{kVp} \mathrm{X-ray} \mathrm{for} \mathrm{measurement}$ of trabecular bone microstructure. Trabecular bone parameters were analyzed as described previously (Cheng et al. 2014). Marrow adipose tissue was visualized and quantified as described (Scheller et al. 2014). Briefly, femurs and tibiae were collected, cleaned and fixed in $4 \%$ PFA for $72 \mathrm{~h}$ at $4^{\circ} \mathrm{C}$. The bones were then washed and decalcified for 18 days in $14 \%$ EDTA at $4^{\circ} \mathrm{C}$. Decalcified bones were stained for lipid with a mixture of equal volumes $2 \%$ osmium tetroxide and 5\% potassium dichromate for $60 \mathrm{~h}$. After washing, the stained bones were imaged using microCT with energy of $55 \mathrm{kVp}$, an integration time of $500 \mathrm{~ms}$ and a maximum isometric voxel size of $10 \mu \mathrm{m}$.

\section{Dynamic histomorphometry}

TR $\beta 147 \mathrm{~F}$ mice and littermate controls were injected intraperitoneally with calcein $(20 \mathrm{mg} / \mathrm{kg}$, Sigma-Aldrich) 6 and 2 days before killing. Tibiae were dissected and fixed in $10 \%$ formalin for 3 days before methylmethacrylate resin embedding and sectioning. Longitudinal sections of the metaphysis were used to measure trabecular bone formation parameters. Calcein labeling was visualized using the Olympus BX60 fluorescence microscope (Olympus Corporation) and analyzed using the OsteoMeasure software (Osteometrics, Inc., Decatur, GA, USA). The mineral apposition rate (MAR), mineralizing surface per bone surface (MS/BS) and bone formation rate per bone surface (BFR/BS) were calculated according to established methods (Parfitt et al. 1987).

\section{Immunoblotting}

Cells were lysed in a buffer containing 0.5\% SDS, $0.1 \% \mathrm{v} / \mathrm{v}$ Triton X-100, $0.25 \%$ sodium deoxycholate, $12.5 \mathrm{mM}$ Tris and $96 \mathrm{mM}$ glycine (pH 8.3). Lysate protein concentrations were determined with a Bradford assay, and equal amounts of protein were run on SDS-PAGE gels for immunoblotting on PVDF membranes. Membranes were probed with antibodies specific for $\beta$-catenin (C7207, Sigma-Aldrich), histone 3 (H0164, Sigma-Aldrich) and $\beta$-actin (A5441, Sigma-Aldrich) before chemiluminescent imaging and quantitation with ImageJ.

\section{Gene expression analysis}

Total RNA was extracted from isolated tissues or cells and synthesized into cDNA for real-time RT-PCR analysis as previously described (Cheng et al. 2012). Relative gene expression levels were examined and the housekeeping gene Ppia was used as an internal control in the PCR reaction. The fold-change compared to control was calculated according to the formula $2^{-\Delta \Delta C t}$. Primer sequences are listed in Table 1.

\section{Statistical analysis}

Results are expressed as mean \pm s.E.M. All experiments were analyzed using Student's t-tests or two-way ANOVA as appropriate ( $\mathrm{R}$ 3.5.3) (https://www.r-project.org/). Differences were considered significant when $P<0.05$.

\section{Results}

\section{TRß147F mice had less trabecular bone}

In previous studies, we reported that thyroid hormonedeficient Tshrhyt/hyt mutant mice had reduced trabecular

Table 1 Primer sequences for real time RT-PCR.

\begin{tabular}{|c|c|c|}
\hline Gene & Forward & Reverse \\
\hline$A / p$ & 5'-ATGGTAACGGGCCTGGCTACA-3' & 5'-AGTTCTGCTCATGGACGCCGT-3' \\
\hline Axin2 & 5'-TTTGGCACAGCTAGAGGAAG-3' & 5'-TGGCTCTTTGTGATCTTCTGG-3' \\
\hline Cyclind1 & 5'-AATGTACTCTGCTTTGCTGAA-3' & 5'-ATGAGACCACTAGAGGTCG-3' \\
\hline Lef1 & 5'-GATCTGGTTGATAGCTGCACTC-3' & 5'-GTAACACCTCAGGTCAAACAGG-3' \\
\hline Osx & 5'-TCСTCTCTGCTTGAGGAAGAAG-3' & 5'-GAGTCCATTGGTGCTTGAGAAG-3' \\
\hline Pat2 & 5'-GTGCCAAGAAGCTGCAGAG-3' & 5'-TGTTGCCTTTGACCAGATGA-3' \\
\hline Pgc1a & 5'-GCAGCCAAGACTCTGTATGG-3' & 5'-TTCCGATTGGTCGCTACACC-3' \\
\hline Ppia & 5'-CCATGGCAAATGCTGGACCA-3' & 5'-TCCTGGACCCAAAACGCTCC-3' \\
\hline Prdm16 & 5'-CAGCACGGTGAAGCCATTC-3' & 5'-GCGTGCATCCGCTTGTG-3' \\
\hline$T c f 7$ & 5'-GCAGGGAAGTGCTGTCTATATC-3' & 5'-CAGCTTTCTCCACTCTACGAAC-3' \\
\hline$T c f 7 / 1$ & 5'-TGTAGGTGATGAGAGGTGTCAG-3' & 5'-GCTACAGTCAAGGACACAAGGT-3' \\
\hline
\end{tabular}


bone mass compared to euthyroid Tshrhyt/+ littermate control mice (Xing et al. 2012). To determine whether $\mathrm{TH}$ effects on trabecular bone mass are mediated through nongenomic TR $\beta-P I 3 K$ signaling, we evaluated the skeletal phenotype of TR $\beta 147 \mathrm{~F}$ mice which have intact genomic TR $\beta$ signaling but disrupted TR $\beta-P I 3 K$ signaling. Using microCT (Fig. 1), we found that female TR $\beta 147 \mathrm{~F}$ mice had less trabecular bone volume adjusted for tissue volume (BV/TV) than littermate controls at the femoral secondary spongiosa ( $20 \%$ reduction, $P<0.05$ ). Male TR $\beta 147 \mathrm{~F}$ mice also had less BV/TV at this site, although the reduction did not reach statistical significance (15\% reduction, $P=0.06)$. This decrease in BV/TV at the femoral secondary spongiosa was explained by significant decreases in trabecular thickness in both sexes (F: 6\% reduction, M: $8 \%$ reduction, both $P<0.05)$. Connectivity density was also reduced in female TR $\beta 147 \mathrm{~F}$ mice $(29 \%$ reduction, $P<0.05)$.

In addition to regulating trabecular bone mass at the secondary spongiosa, we had found that the increase in $\mathrm{TH}$ levels during the second week of postnatal life in mice is essential for initiation and progression of secondary ossification and that trabecular bone volume at the epiphysis was severely compromised in thyroid hormonedeficient mice (Xing et al. 2014, 2016). Consistent with a role for TR $\beta$ nongenomic signaling, we found a significant reduction in $\mathrm{BV} / \mathrm{TV}$ at the tibial epiphysis (9\% reduction, $P<0.05$; Fig. 2) in both female and male TR $\beta 147 \mathrm{~F}$ mice.
There were significant decreases in trabecular number (F: 9\% reduction, $\mathrm{M}$ : $8 \%$ reduction, both $P<0.05$ ) and trabecular thickness (F: 9\% reduction, $\mathrm{M}: 17 \%$ reduction, both $P<0.05)$ in the mutant mice. However, trabecular separation was increased (F: $12 \%$ increase, $P<0.05$, M: $8 \%$ increase, $P=0.05$ ) in the mutant mice compared to control littermates.

Similarly, male mutant TR $\beta 147 \mathrm{~F}$ mice had less trabecular bone at lumbar vertebra L5 (Fig. 3). BV/TV was reduced 9\% $(P<0.05)$, and this decrease was accompanied by a $30 \%$ reduction in connectivity density, $9 \%$ reduction in trabecular number, and 11\% increase in trabecular separation (all $P<0.05$ ).

To determine if TR $\beta$ nongenomic signaling also contributed to cortical bone changes, we measured cortical bone volume at the mid-diaphysis of mutant and control mice (Fig. 4). While bone length was not affected in mutant mice, cortical BV adjusted for TV was slightly less in both the femur and tibia of mutant mice, although this reduction was only significant in the femurs of male TR $\beta 147$ F mice (5\% reduction, $P<0.05$ ).

\section{Trabecular bone formation rate is decreased in TRß147F mice}

Dynamic histomorphometry was performed on calcein double-labeled femurs from TR $\beta 147 \mathrm{~F}$ mice and littermate
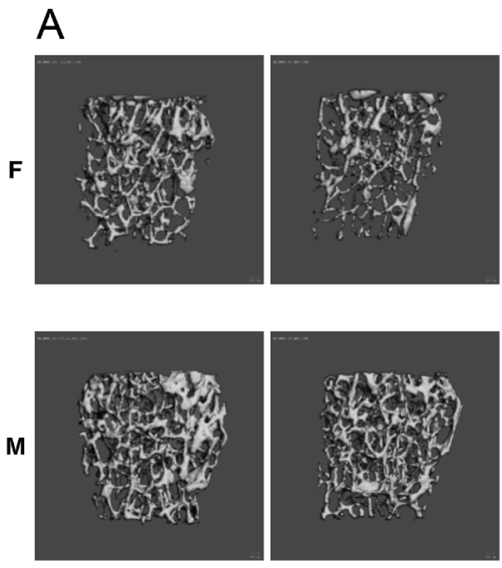

Het

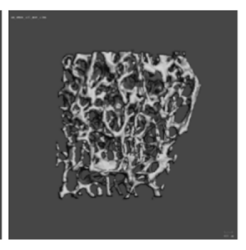

Mut

\section{Femoral Trabecular}
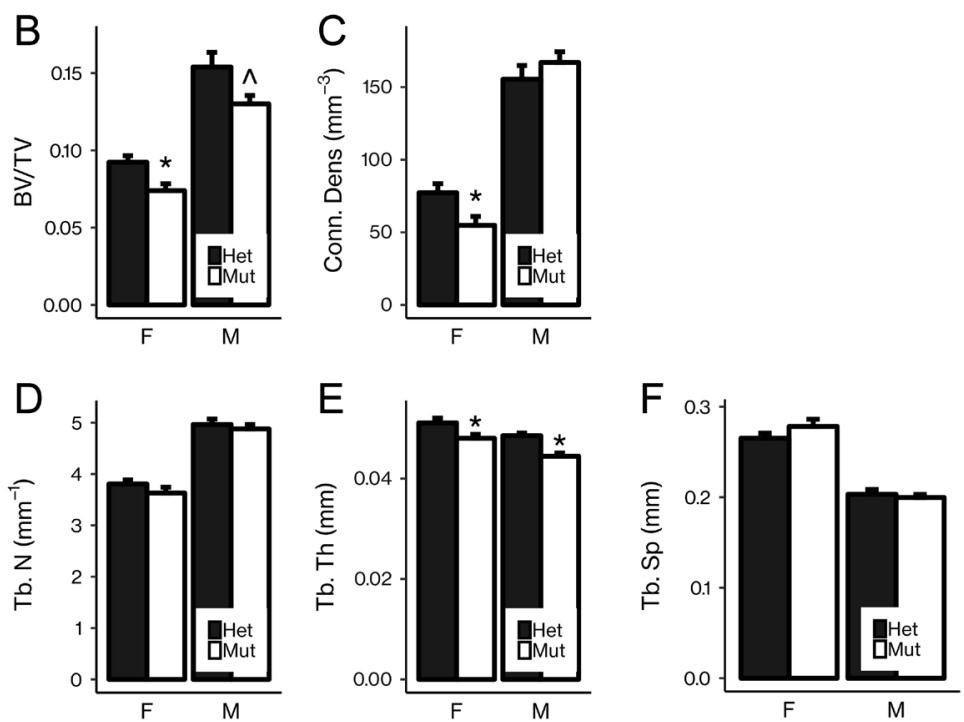

Figure 1

TR $1477 \mathrm{~F}$ mutant mice have decreased femoral trabecular bone mass. (A) Representative microCT images of femoral trabecular bone from control (Het) and TR 147 F (Mut) mice. Quantitated (B) bone volume adjusted for tissue volume (BV/TV), (C) connectivity density (Conn.Dens, mm-3), (D) trabecular number (Tb.N, mm-1), (E) trabecular thickness (Tb.Th, mm) and (F) trabecular separation (Tb.Sp, mm), separated by sex (F: female, M: male). Results are presented as mean \pm S.E.M. ${ }^{*} P<0.05,{ }^{\wedge} P<0.1$ vs Het, $n=5-8$. 

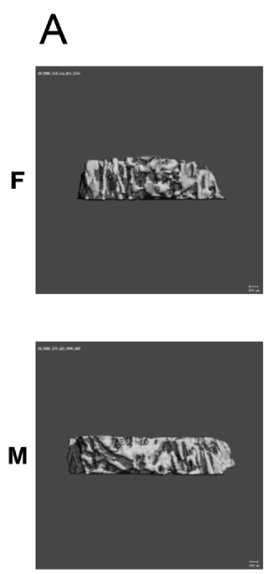

Het
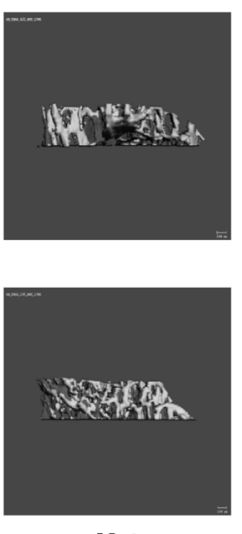

Mut

Tibial Epiphyseal
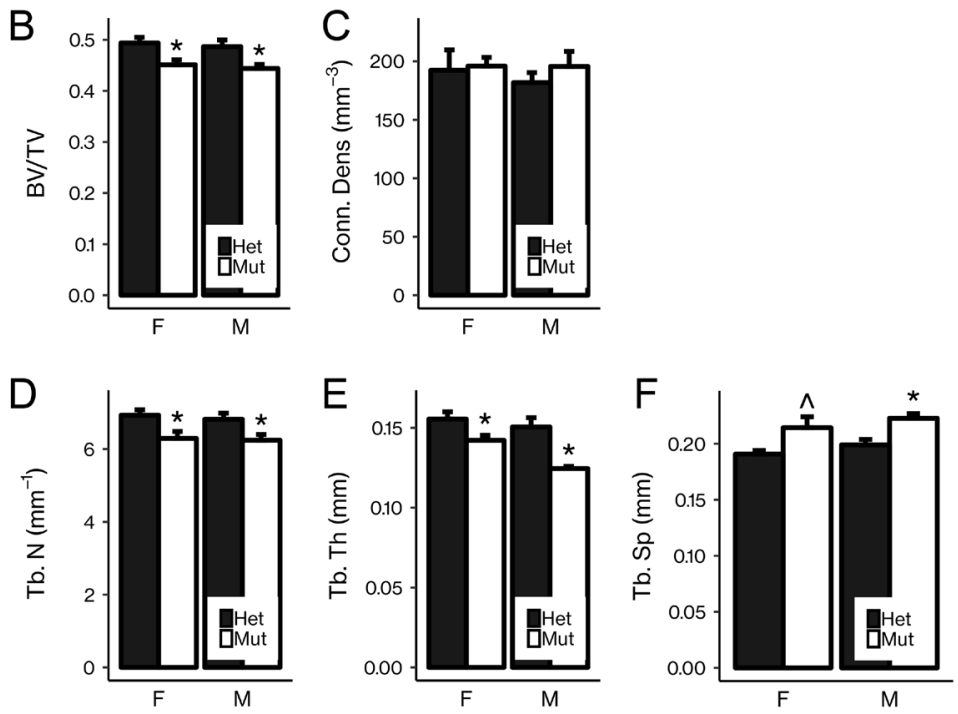

Figure 2

TRß147F mutant mice have decreased tibial epiphyseal bone mass. (A) Representative microCT images of tibial trabecular bone from control (Het) and TRß147F (Mut) mice. Quantitated (B) bone volume adjusted for tissue volume (BV/TV), (C) connectivity density (Conn.Dens, mm³), (D) trabecular number (Tb. N, mm-1), (E) trabecular thickness (Tb.Th, mm), and (F) trabecular separation (Tb.Sp, mm), separated by sex (F: female, M: male). Results are presented as mean \pm S.E.M. $* P<0.05, \wedge p<0.1$ vs Het, $n=5-7$.

controls (Fig. 5). While MAR was not affected, TR $\beta 147 \mathrm{~F}$ mice had significantly less mineralizing surface per bone surface (MS/BS) than controls (29\% reduction, $P<0.05)$. Thus, the overall trabecular bone formation rate adjusted for bone surface (BFR/BS) was decreased in TR $\beta 147 \mathrm{~F}$ mice ( $27 \%$ reduction, $P<0.05$ ). Since MAR was unaffected, the reduction in BFR in mutant mice appears to be due to a reduced number of fully differentiated osteoblasts, not a reduction in osteoblast activity.

\section{TRß147F mice had more bone marrow adiposity}

Since we have previously shown that TR $\beta$ signaling regulates bone marrow adiposity (Lindsey \& Mohan 2017), we investigated whether disruption of nongenomic TR $\beta$-PI3K signaling had an effect on bone marrow adipose tissue. Tibiae from TR $\beta 147 \mathrm{~F}$ and littermate control mice were decalcified and stained with osmium tetroxide to allow for marrow adipose tissue measurement by microCT (Fig. 6). We found significantly more marrow adipose tissue in the proximal, regulated region of the tibia of TR $\beta 147 \mathrm{~F}$ mice (20\% increase, $P<0.05)$, although this change was only significant in female mice, not males (data not shown).
TR $\beta$ agonist requires nongenomic TR $\beta-P I 3 K$ signaling to increase $A l p$ and Osx expression in osteoblasts

To evaluate the effect of nongenomic TR $\beta$-PI3K signaling on osteoblast differentiation, primary calvarial osteoblasts were isolated from TR $\beta 147 \mathrm{~F}$ mice and littermate controls. Osteoblasts were grown in culture and treated with TR $\beta$ specific agonist MGL-3196 or GC-1. In control osteoblasts, treatment with the TR $\beta$ agonist induced 1.7-fold and 1.3-fold increases $(P<0.05)$ in mRNA levels of key osteoblast differentiation markers alkaline phosphatase $(A l p)$ and osterix (Os $x$ ), respectively (Fig. 7A). However, TR $\beta$ agonists did not increase Alp or Osx expression in osteoblasts from TR $\beta 147 \mathrm{~F}$ mice, suggesting that the ability of $\mathrm{TH}$ to induce osteoblast differentiation depends at least in part on nongenomic TR $\beta-P I 3 K$ signaling. To demonstrate that genomic TR $\beta$ signaling is still active in osteoblasts from TR $\beta 147 \mathrm{~F}$ mice, we measured the expression of insulin-like growth factor I (Igf1) and Indian hedgehog (Ihh) and found increases after TR $\beta$ agonist treatment in both control and mutant cells (Fig. 7B), although the increase in $I h h$ in mutant osteoblasts did not quite reach statistical significance $(P=0.08)$. Interestingly, TR $\beta$ agonist also increased expression of brown-like adipose tissue marker genes Pat2, Pgc1a and Prdm16 (Fig. 7C), 
Lumbar Trabecular
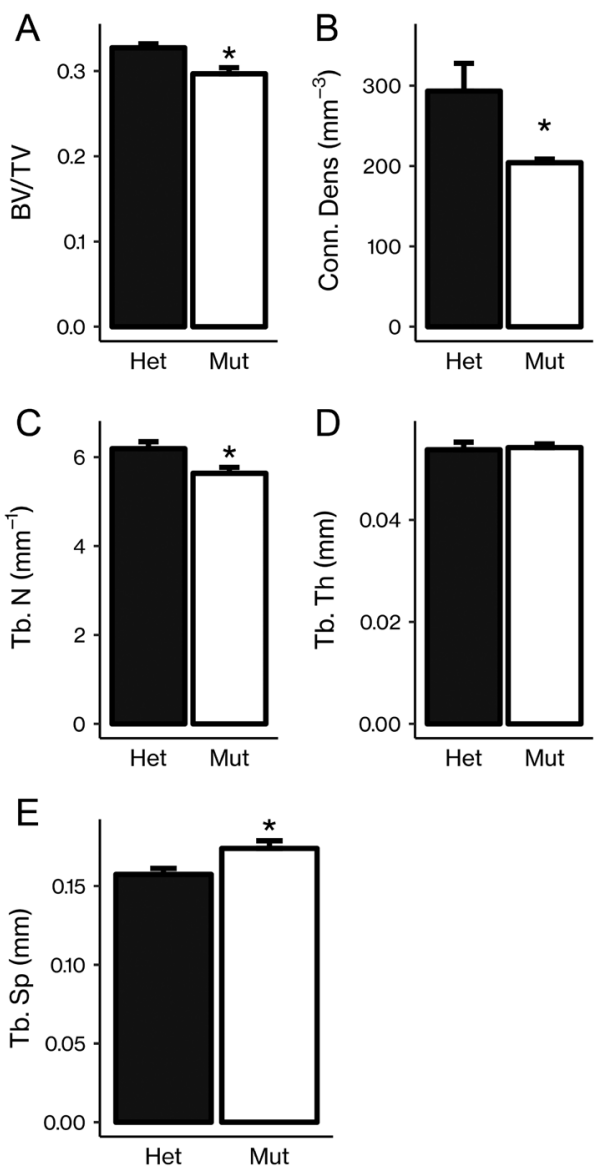

Figure 3

TR $1477 \mathrm{~F}$ mutant mice have decreased trabecular bone mass at lumbar vertebra L5. Quantitated (A) bone volume adjusted for tissue volume (BV/TV), (B) connectivity density (Conn.Dens, $\mathrm{mm}^{-3}$ ), (C) trabecular number (Tb.N, $\left.\mathrm{mm}^{-1}\right),(\mathrm{D})$ trabecular thickness (Tb.Th, $\mathrm{mm}$ ) and (E) trabecular separation (Tb.Sp, mm) in male control (Het) and TR $\beta 147 \mathrm{~F}$ (Mut) mice. Results are presented as mean \pm S.E.M. ${ }^{*} P<0.05$ vs Het, $n=4-6$.

which is consistent with our previous report that activation of TR $\beta$ increased expression of brown-like adipose tissue marker genes in marrow adipose tissue (Lindsey \& Mohan 2017).

\section{$\beta$-Catenin signaling in osteoblasts was upregulated by TR $\beta$ agonist treatment}

Since PI3K signaling is known to activate $\beta$-catenin, particularly in the context of thyroid cancer (SastrePerona et al. 2016), and since $\beta$-catenin signaling is important for osteoblast differentiation (Moorer \& Riddle 2018), we hypothesized that the ability of nongenomic TR $\beta$-PI3K signaling to induce osteoblast differentiation may be mediated via $\beta$-catenin signaling. To test this hypothesis, we treated MC3T3-E1 pre-osteoblasts with
TR $\beta$-specific agonist GC-1 and measured cytoplasmic and nuclear levels of $\beta$-catenin protein by immunoblot (Fig. 8A). While we did not detect a significant change in $\beta$-catenin protein levels after GC-1 treatment, we found significant increases in mRNA expression levels of $\beta$-catenin target genes Cyclind1, Lef1 and Tcf7 after GC-1 treatment (Fig. 8B). Furthermore, TR $\beta$ agonist treatment induced a 1.6-fold increase $(P<0.05)$ in $\beta$-catenin target gene Axin2 in calvarial osteoblasts derived from control mice, but this effect was blocked in osteoblasts from TR $\beta 147 \mathrm{~F}$ mice (Fig. 8C).

\section{Discussion}

Many of the hormones which are classically thought of as exerting their effects via nuclear receptors which act by genomic regulation of gene expression have more recently been observed to have additional rapid effects on the cell mediated by those same receptors acting in the cytoplasm. Significant study has been devoted to these nongenomic mechanisms of steroid hormone signaling (Kousteni et al. 2001, Losel et al. 2003, Lösel \& Wehling 2003, Hammes \& Levin 2011). For example, membrane estrogen receptors have been shown to contribute to cardiovascular health, and cytoplasmic estrogen receptors are known to regulate proliferation, migration and invasion of cancer cells (Hammes \& Levin 2011). However, more recent studies have shown that TH can also have rapid cellular effects which occur too quickly to be explained by changes in gene expression (Davis et al. 2008, 2018). In the search for mechanisms which would explain these rapid, nongenomic effects, PI3K was implicated as playing a role (Incerpi et al. 2002, Storey et al. 2002, 2006, Lei et al. 2004). While both TH receptor types TR $\alpha$ (Simoncini et al. 2000, Hiroi et al. 2006) and TR $\beta$ (Saelim et al. 2004, Cao et al. 2005, Moeller et al. 2005, Verga Falzacappa et al. 2007) are known to associate with PI3K, it has been shown that TR $\beta$ is necessary for at least one of the specific PI3K-dependent effects of TH (Storey et al. 2006).

In particular, a previous study by Martin et al. (2014) showed that TR $\beta$ forms a complex with the p85 subunit of PI3K and Src family kinase Lyn. Incubation with TH led to the activation of PI3K signaling, and pharmacological inhibition of either PI3K or Lyn blocked that PI3K activation. Furthermore, mutation of key tyrosine residues in TR $\beta$ which prevented the interaction between TR $\beta$ and either p85 or Lyn also blocked the ability of TH to activate PI3K signaling. Creation of the TR $\beta 147 \mathrm{~F}$ mice in which tyrosine 147 of TR $\beta 1$ is replaced by a phenylalanine, 
Endocrinology bone

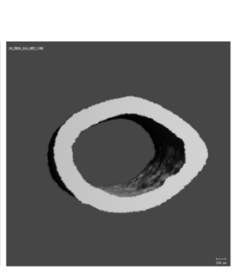

C
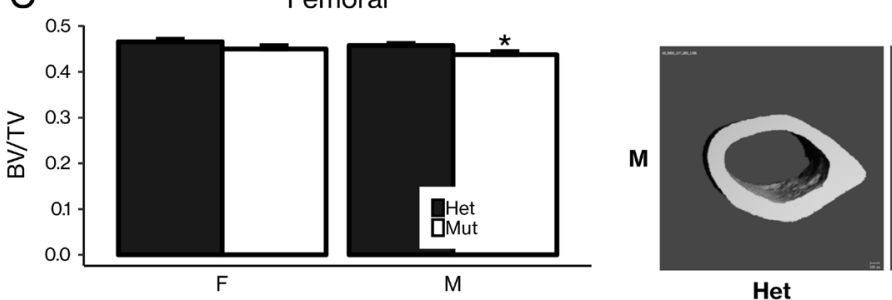

Het

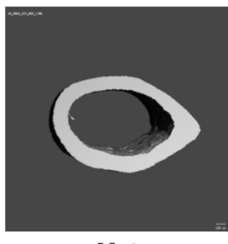

Mut

\section{Figure 4}

TRß147F mutant mice have minimal changes in cortical bone mass. (A) Femur length from control (Het) and TR $\beta 147 \mathrm{~F}$ (Mut) mice, separated by sex. (B) Representative microCT images of femoral cortical bone at the mid-diaphysis. Quantitated cortical bone volume adjusted for tissue volume (BV/TV) at the (C) femur and (D) tibia of control (Het) and TR $\beta 147 \mathrm{~F}$ (Mut) mice, separated by sex (F: female, M: male). Results are presented as mean \pm S.E.M. ${ }^{*} P<0.05$ vs Het, $n=5-8$. preventing the interaction of TR $\beta$ with Lyn and thus disrupting the nongenomic TR $\beta$-PI3K signaling pathway while leaving the genomic TR $\beta$ pathway intact, allowed for the first time in vivo investigation of the effects of this nongenomic mechanism. In that particular study, TR $\beta 147 \mathrm{~F}$ mice were found to have significantly impaired maturation of synapses in the hippocampus (Martin et al. 2014).

Since we have previously shown that $\operatorname{TR} \beta$ mediates at least some of TH's effects on skeletal development, we wondered whether part of that effect might be via nongenomic TR $\beta-P I 3 K$ signaling. To investigate the effects of nongenomic TR $\beta-\mathrm{PI} 3 \mathrm{~K}$ signaling in vivo, we evaluated the skeletal phenotype of TR $\beta 147 \mathrm{~F}$ mice. Using microCT, we found that disruption of nongenomic TR $\beta$-PI3K signaling led to significant decreases in trabecular bone at the femoral secondary spongiosa, tibial epiphysis and lumbar vertebra, suggesting that this nongenomic pathway is required for $\mathrm{TH}$ to have its full effect during skeletal development. Small differences were observed in the skeletal phenotypes of female vs male TR $\beta 147 \mathrm{~F}$ mice; although similar trends were observed in both sexes, more studies are needed to determine whether the differences in the magnitude of the observed phenotypes are due

\section{Dynamic Histomorphometry}

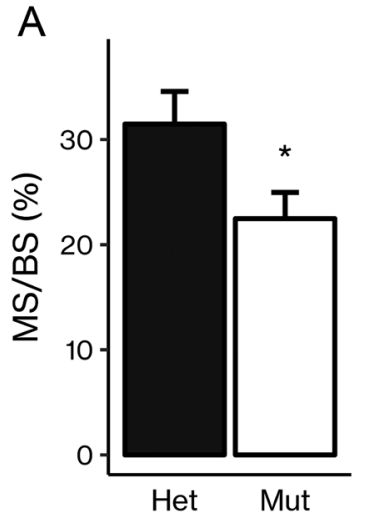

https://joe.bioscientifica.com https://doi.org/10.1530/JOE-19-0172
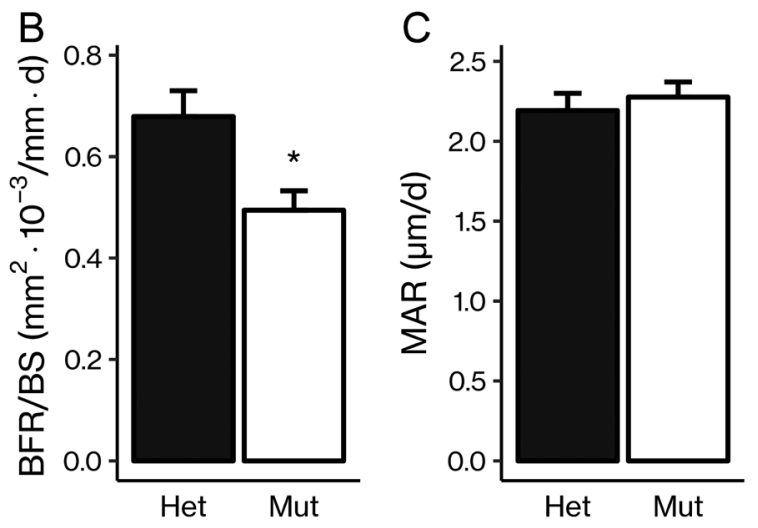

\section{Figure 5}

Trabecular bone formation rate is decreased in TRß147F mice. Dynamic histomorphometry was performed on calcein double-labeled femurs from control (Het) and TRß147F (Mut) mice. Quantitated (A) mineralizing surface per bone surface (MS/BS, $\%)$, (B) bone formation rate adjusted for bone surface (BFR/BS, $\mathrm{mm}^{2} \times 10^{-3} / \mathrm{mm} \times$ day), and (C) mineral apposition rate (MAR, $\mu \mathrm{m} /$ day). Results are presented as mean \pm S.E.M. ${ }^{*} P<0.05$ vs Het, $n=6-11$. 
A

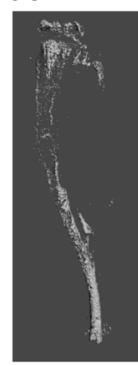

Het

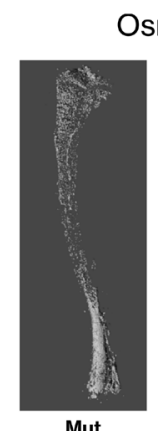

Mut
Osmium microCT

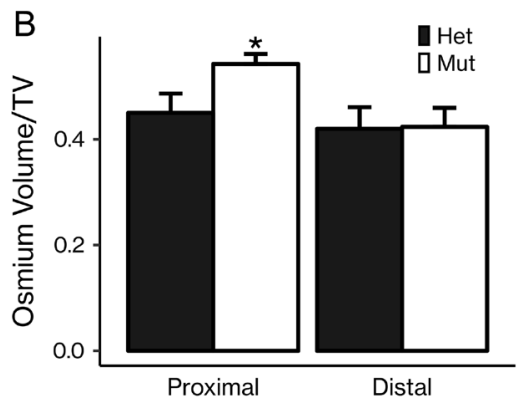

Figure 6

Disrupted nongenomic TR $\beta 1$ signaling increased bone marrow adiposity. (A) Representative microCT images of decalcified, osmium tetroxidestained tibiae from control (Het) and TR $147 \mathrm{~F}$ (Mut) mice. (B) Quantitated osmium tetroxide volume adjusted for tissue volume in the regulated, proximal region of the tibia and the constitutive, distal region of the tibia in female mice. Results are presented as mean \pm S.E.M. $* P<0.05$ vs Het, $n=4-7$.

to potential interaction of the nongenomic TR $\beta-P I 3 K$ pathway with other signaling pathways (e.g., estrogen signaling). We have not observed sex-specific differences in skeletal phenotype in our previous studies of $\mathrm{TH}$ regulation of bone formation; however, some differences have been reported in the skeletal phenotype of congenital hypothyroidism (Van Vliet et al. 2003), and the rates of sexual dysfunction caused by TH derangement may be different between the sexes (Bates et al. 2018), suggesting the possibility of interaction between thyroid and sex steroid signaling. Indeed, $\mathrm{TH}$ and the sex steroids all signal via nuclear receptors and have overlapping genomic and nongenomic effects; in fact, some nongenomic actions of TH have been shown to mimic the effects of estrogen in some tumor cells (Hammes \& Davis 2015), suggesting a possible mechanism for sex-specific effects of TH.

Using dynamic histomorphometry, we determined that the mutant TR $\beta 147 \mathrm{~F}$ mice have a significantly decreased bone formation rate adjusted for bone surface.

This decrease in BFR/BS was due to a reduction in mineralizing surface per bone surface; MAR was unchanged, suggesting that a reduction in the number of fully differentiated, mature osteoblasts, not a reduction in osteoblast activity, was responsible for the overall decrease in bone formation. In our studies, we used heterozygous littermates as control mice for comparison to mutant mice. The use of heterozygous mice as controls should not weaken our conclusions about the effect of the TR $\beta 147 \mathrm{~F}$ mutation on bone since, if anything, we would expect the phenotypic difference to be larger if WT mice were used as controls.

In order to explore the mechanism of nongenomic TR $\beta$-PI3K signaling's effect on the skeleton, we treated primary osteoblasts from TR $\beta 147 \mathrm{~F}$ mice with TR $\beta$-specific agonists MGL-3196 or GC- 1 . The fact that these TR $\beta$ agonists were able to upregulate mRNA expression of $A l p$ and Osx, crucial markers of osteoblast differentiation, in control osteoblasts but not in mutant TR $\beta 147 \mathrm{~F}$ osteoblasts suggests that nongenomic TR $\beta$-PI3K signaling is important for the ability of TH to induce osteoblast differentiation.

We next hypothesized that the nongenomic TR $\beta$-PI3K signaling pathway could be regulating osteoblast differentiation via activation of $\beta$-catenin signaling, since $\beta$-catenin has been shown to be activated by PI3K in thyroid cancer and $\beta$-catenin is a key regulator of osteoblast proliferation and differentiation. While we did not detect a significant change in protein levels of $\beta$-catenin in MC3T3-E1 pre-osteoblasts after treatment with TR $\beta$ agonist GC-1, we did find significant increases in mRNA expression of $\beta$-catenin target genes in MC3T3-E1 pre-osteoblasts after treatment with TR $\beta$ agonist GC-1. This upregulation of $\beta$-catenin signaling markers in response to GC- 1 suggests that $\beta$-catenin may mediate at least some of the nongenomic TR $\beta-P I 3 K$ effect on osteoblasts. Furthermore, TR $\beta$-specific agonist MGL-3196 was able to upregulate Axin2, a $\beta$-catenin target gene,
A

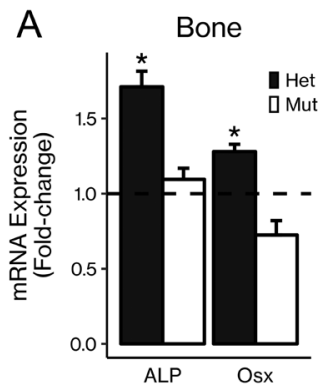

B

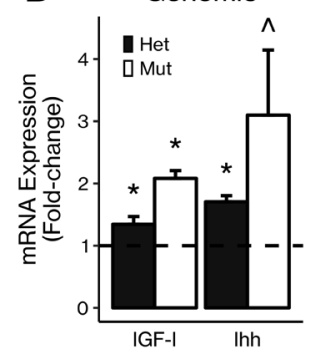

C

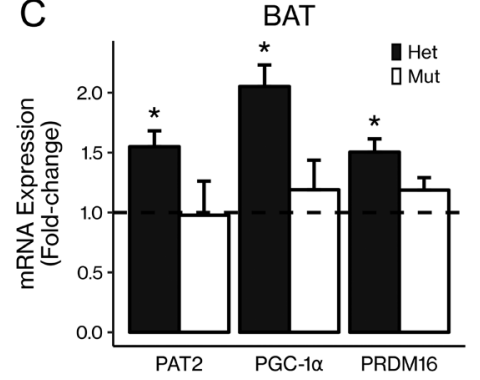

Figure 7

Nongenomic TR $\beta 1$ signaling is required for upregulation of marker genes. Primary calvarial osteoblasts from control (Het) or TR $\beta 147 \mathrm{~F}$ (Mut) mice were treated in vitro with TR $\beta 1$-specific agonist MGL-3196 or GC-1 for 3 days before RNA extraction and RT-qPCR with primers specific for (A) osteoblast differentiation markers alkaline phosphatase (A/p) and osterix (Osx), (B) genomic TR $\beta$ response genes insulin-like growth factor 1 (Igf1) and Indian hedgehog (Ihh) and (C) brownlike adipose markers Pat2, Pgc1a and Prdm16. Results are presented as mean fold-change \pm S.E.M. $\star P<0.05,{ }^{\wedge} P<0.1$ vs vehicle, $n=2-4$. 


\section{Figure 8}

$\beta$-catenin signaling was upregulated by activation of TR $\beta 1$. MC3T3-E1 pre-osteoblasts and calvarial osteoblasts from control (Het) and TR $\beta 147 \mathrm{~F}$ (Mut) mice were treated with TR $\beta 1$-specific agonist GC-1 or MGL-3196 for three days. (A) Cytoplasmic and nuclear protein were extracted from MC3T3-E1 cells, and $\beta$-catenin protein expression (normalized to $\beta$-actin and histone 3 ) was measured by immunoblotting. (B) RNA was extracted from MC3T3-E1 cells, and RT-qPCR was performed with primers specific for $\beta$-catenin target genes Cyclind1, Lef1, Tcf7 and Tcf7/1. (C) RNA was extracted from Het and Mut calvarial osteoblasts, and RT-qPCR was performed with primers specific for $\beta$-catenin target gene Axin2. Results are presented as mean (A) \% of control or $(B, C)$ fold-change vs control \pm S.E.M. $* P<0.05$, $\wedge P<0.1, n=3-4$ in calvarial osteoblasts derived from control but not from TR $\beta 147 \mathrm{~F}$ mice, lending further support to the hypothesis that $\beta$-catenin signaling may mediate nongenomic TR $\beta-P I 3 K$ effects. Further studies are needed to explore and evaluate the many other candidate pathways which could mediate these effects; RNA-seq followed by pathway analysis of mutant TR $\beta 147 \mathrm{~F}$ vs control osteoblasts may be an appropriate follow-up study.

Since we have previously shown that TR $\beta$ signaling can lead to a decrease in the amount of bone marrow adipose tissue in mice (Lindsey \& Mohan 2017), we wondered whether nongenomic TR $\beta-P I 3 K$ signaling contributes to this effect on marrow adiposity. Using microCT on osmium tetroxide-stained tibiae to examine the bone marrow adiposity phenotype of TR $\beta 147 \mathrm{~F}$ mice, we found that mutant mice had more marrow adiposity in the proximal, regulated region of the tibia than control mice, suggesting that nongenomic TR $\beta$ signaling does indeed contribute to the ability of TR $\beta$ to decrease marrow

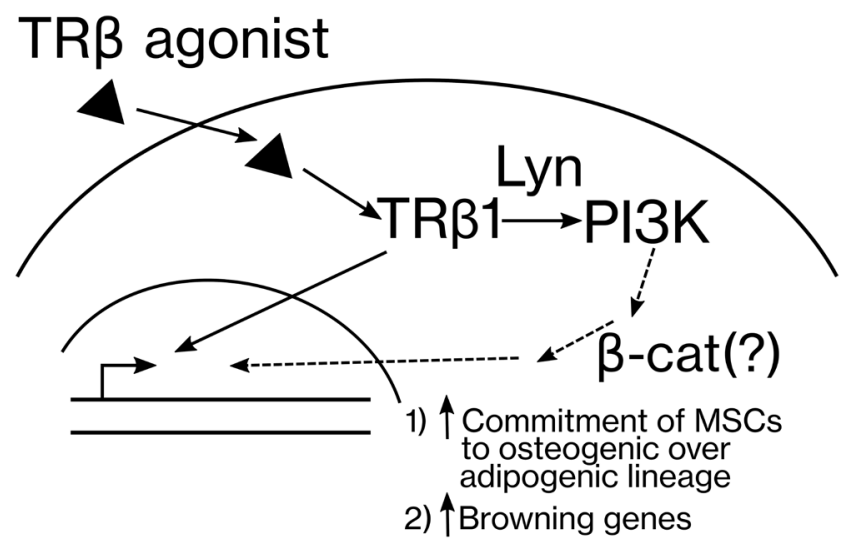

Figure 9

Proposed model of TR $\beta$ regulation of osteoblasts via both traditional genomic signaling and nongenomic TR $\beta-P I 3 K$ signaling.

(c) 2019 Society for Endocrinology Published by Bioscientifica Ltd. Printed in Great Britain adiposity. In our previous study, we proposed the so-called 'browning' of marrow adipose tissue as a potential mechanism contributing to this negative regulation of marrow adiposity by TR $\beta$ signaling; since brown-like adipose tissue is much more metabolically active and contains smaller lipid droplets than white adipose tissue, an increase in the proportion of brown-like adipose tissue in the marrow could explain the decrease in MAT volume caused by $\mathrm{TH}$. Indeed, we were able to demonstrate the upregulation of brown-like adipose tissue marker genes in response to the activation of $\operatorname{TR} \beta$ signaling (Lindsey \& Mohan 2017). The fact that we and others have been unable to show an upregulation of UCP1 expression in marrow adipose tissue suggests that marrow adipose tissue does not become true brown/beige adipose tissue but may constitute a unique type of adipose tissue which shares some characteristics of both white and brown adipose tissue. In any case, we found that treatment of control but not TR $\beta 147 \mathrm{~F}$ osteoblasts with TR $\beta$-specific agonist MGL-3196 upregulated the expression of brown-like adipose tissue marker genes Pat2, Pgc1a and Prdm16, suggesting that the TR $\beta$ effect on marrow adipose tissue phenotype may require nongenomic TR $\beta-P I 3 K$ signaling. Since only female TR $\beta 147 \mathrm{~F}$ mice had increased marrow adipose tissue, however, further study is needed to determine the mechanisms of this effect and whether they might interact with other sex-specific physiological processes.

In conclusion, we have shown that nongenomic TR $\beta$-PI3K signaling is a contributor to TH's effects on skeletal development and osteoblast differentiation (Fig. 9). While our studies implicate involvement of nongenomic TR $\beta$ signaling in regulating bone and MAT phenotypes, it remains to be determined whether all of the effects of TR $\beta$ are mediated through this nongenomic 
pathway. To this end, we need to compare the skeletal and MAT phenotypes of mice with disruption of both genomic and nongenomic signaling to see if the observed changes in BMD and MAT phenotypes in these mice are comparable to what we found with disruption of nongenomic signaling only. This novel mechanism represents a new possible set of therapeutic targets for the development of osteoporosis treatments. Further investigation of the downstream mediators of this signaling pathway is needed to elucidate the potential targets for these urgently needed therapies which will reduce the economic and quality-of-life burden of this debilitating disease. In addition, we have demonstrated that TR $\beta$-PI3K signaling regulates marrow adipose tissue. Thus, targeting this nongenomic signaling pathway has the potential to affect adipose tissue metabolism. Future studies are needed to determine the mechanisms of this effect and the extent to which this pathway could be targeted more broadly to treat obesity and other metabolic diseases.

\section{Declaration of interest}

The authors declare that there is no conflict of interest that could be perceived as prejudicing the impartiality of the research reported.

\section{Funding}

This work was supported by National Institutes of Health grants R01 AR048139 to S M, Senior Research Career Scientist award to S M from the US Department of Veterans Affairs, and 2 R25 GM060507 to the Loma Linda University Center for Health Disparities and Molecular Medicine/ Initiative for Maximizing Student Development.

\section{Author contribution statement}

R C L and S M designed experiments and performed data analysis and interpretation. R C L and C G performed experiments. S M and R C L wrote the manuscript. S M conceived and oversaw the entire the project.

\section{Acknowledgments}

The authors would like to thank Nancy Lowen, Heather Watt and Subhashri Das for their expert technical assistance. In addition, the authors thank Dr David Armstrong for providing the TR $\beta 147 \mathrm{~F}$ mouse strain. All work was performed with the facilities provided by the US Department of Veterans Affairs in Loma Linda, CA.

\section{References}

Aghajanian P, Xing W, Cheng S \& Mohan S 2017 Epiphyseal bone formation occurs via thyroid hormone regulation of chondrocyte to osteoblast transdifferentiation. Scientific Reports 7 10432. (https://doi. org/10.1038/s41598-017-11050-1)
Bates JN, Kohn TP \& Pastuszak AW 2018 Effect of thyroid hormone derangements on sexual function in men and women. Sexual Medicine Reviews [epub]. (https://doi.org/10.1016/j.sxmr.2018.09.005)

Cao X, Kambe F, Moeller LC, Refetoff S \& Seo H 2005 Thyroid hormone induces rapid activation of Akt/protein kinase B-mammalian target of rapamycin-p70s6K cascade through phosphatidylinositol 3-kinase in human fibroblasts. Molecular Endocrinology 19 102-112. (https://doi. org/10.1210/me.2004-0093)

Cheng S, Zhao SL, Nelson B, Kesavan C, Qin X, Wergedal J, Mohan S \& Xing W 2012 Targeted disruption of ephrin B1 in cells of myeloid lineage increases osteoclast differentiation and bone resorption in mice. PLOS ONE 7 e32887. (https://doi.org/10.1371/journal. pone.0032887)

Cheng S, Xing W, Pourteymoor S \& Mohan S 2014 Conditional disruption of the prolyl hydroxylase domain-containing protein 2 (Phd2) gene defines its key role in skeletal development. Journal of Bone and Mineral Research 29 2276-2286. (https://doi.org/10.1002/ jbmr.2258)

Davis PJ, Leonard JL \& Davis FB 2008 Mechanisms of nongenomic actions of thyroid hormone. Frontiers in Neuroendocrinology 29 211-218. (https://doi.org/10.1016/j.yfrne.2007.09.003)

Davis PJ, Leonard JL, Lin H-Y, Leinung M \& Mousa SA 2018 Molecular basis of nongenomic actions of thyroid hormone. Vitamins and Hormones 106 67-96. (https://doi.org/10.1016/bs.vh.2017.06.001)

Drake MT \& Khosla S 2019 Sex steroids and the pathogenesis of osteoporosis. In Primer on the Metabolic Bone Diseases and Disorders of Mineral Metabolism, 9th ed., pp 412-418. Ed JP Bilezikian. Hoboken, NJ, USA: Wiley-Blackwell. (https://doi.org/10.1002/9781119266594.ch52)

Forrest D, Sjöberg M \& Vennström B 1990 Contrasting developmental and tissue-specific expression of alpha and beta thyroid hormone receptor genes. EMBO Journal 9 1519-1528. (https://doi. org/10.1002/j.1460-2075.1990.tb08270.x)

Gouveia CHA, Miranda-Rodrigues M, Martins GM \& Neofiti-Papi B 2018 Thyroid hormone and skeletal development. Vitamins and Hormones 106 383-472. (https://doi.org/10.1016/bs.vh.2017.06.002)

Hammes SR \& Davis PJ 2015 Overlapping nongenomic and genomic actions of thyroid hormone and steroids. Best Practice and Research Clinical Endocrinology and Metabolism 29 581-593. (https://doi. org/10.1016/j.beem.2015.04.001)

Hammes SR \& Levin ER 2011 Minireview: recent advances in extranuclear steroid receptor actions. Endocrinology 152 4489-4495. (https://doi. org/10.1210/en.2011-1470)

Hiroi Y, Kim H-H, Ying H, Furuya F, Huang Z, Simoncini T, Noma K, Ueki K, Nguyen N-H, Scanlan TS, et al. 2006 Rapid nongenomic actions of thyroid hormone. PNAS 103 14104-14109. (https://doi. org/10.1073/pnas.0601600103)

Incerpi S, De Vito P, Luly P, Spagnuolo S \& Leoni S 2002 Short-term effects of thyroid hormones and 3,5-diiodothyronine on membrane transport systems in chick embryo hepatocytes. Endocrinology 143 1660-1668. (https://doi.org/10.1210/endo.143.5.8767)

Kousteni S, Bellido T, Plotkin LI, O'Brien CA, Bodenner DL, Han L, Han K, DiGregorio GB, Katzenellenbogen JA, Katzenellenbogen BS, et al. 2001 Nongenotropic, sex-nonspecific signaling through the estrogen or androgen receptors: dissociation from transcriptional activity. Cell 104 719-730. (https://doi.org/10.1016/S0092-8674(01)00268-9)

Lei J, Mariash CN \& Ingbar DH 2004 3,3',5-Triiodo-L-thyronine up-regulation of $\mathrm{Na}, \mathrm{K}-\mathrm{ATPa}$ activity and cell surface expression in alveolar epithelial cells is Src kinase- and phosphoinositide 3-kinasedependent. Journal of Biological Chemistry 279 47589-47600. (https:// doi.org/10.1074/jbc.M405497200)

Lindsey RC \& Mohan S 2017 Thyroid hormone acting via TR $\beta$ induces expression of browning genes in mouse bone marrow adipose tissue. Endocrine 56 109-120. (https://doi.org/10.1007/s12020-017-1265-x)

Lindsey RC, Aghajanian P \& Mohan S 2018 Thyroid hormone signaling in the development of the endochondral skeleton. Vitamins and Hormones 106 351-381. (https://doi.org/10.1016/bs.vh.2017.06.003) 
Lösel R \& Wehling M 2003 Nongenomic actions of steroid hormones. Nature Reviews Molecular Cell Biology 4 46-55. (https://doi. org/10.1038/nrm1009)

Losel RM, Falkenstein E, Feuring M, Schultz A, Tillmann H-C, RossolHaseroth K \& Wehling M 2003 Nongenomic steroid action: controversies, questions, and answers. Physiological Reviews $\mathbf{8 3}$ 965-1016. (https://doi.org/10.1152/physrev.00003.2003)

Martin NP, Marron Fernandez de Velasco E, Mizuno F, Scappini EL, Gloss B, Erxleben C, Williams JG, Stapleton HM, Gentile S \& Armstrong DL 2014 A rapid cytoplasmic mechanism for PI3 kinase regulation by the nuclear thyroid hormone receptor, TR $\beta$, and genetic evidence for its role in the maturation of mouse hippocampal synapses in vivo. Endocrinology 155 3713-3724. (https://doi. org/10.1210/en.2013-2058)

Miyakoshi N, Richman C, Kasukawa Y, Linkhart TA, Baylink DJ \& Mohan S 2001 Evidence that IGF-binding protein-5 functions as a growth factor. Journal of Clinical Investigation 107 73-81. (https://doi. org/10.1172/JCI10459)

Moeller LC, Dumitrescu AM \& Refetoff S 2005 Cytosolic action of thyroid hormone leads to induction of hypoxia-inducible factor-1alpha and glycolytic genes. Molecular Endocrinology 19 2955-2963. (https://doi. org/10.1210/me.2004-0542)

Moorer MC \& Riddle RC 2018 Regulation of osteoblast metabolism by Wnt signaling. Endocrinology and Metabolism 33 318-330. (https://doi. org/10.3803/EnM.2018.33.3.318)

Parfitt AM, Drezner MK, Glorieux FH, Kanis JA, Malluche H, Meunier PJ, Ott SM \& Recker RR 1987 Bone histomorphometry: standardization of nomenclature, symbols, and units. Report of the ASBMR Histomorphometry Nomenclature Committee. Journal of Bone and Mineral Research 2 595-610. (https://doi.org/10.1002/jbmr.5650020617)

Saelim N, John LM, Wu J, Park JS, Bai Y, Camacho P \& Lechleiter JD 2004 Nontranscriptional modulation of intracellular $\mathrm{Ca} 2+$ signaling by ligand stimulated thyroid hormone receptor. Journal of Cell Biology 167 915-924. (https://doi.org/10.1083/jcb.200409011)

Sastre-Perona A, Riesco-Eizaguirre G, Zaballos MA \& Santisteban P 2016 $\beta$-catenin signaling is required for RAS-driven thyroid cancer through PI3K activation. Oncotarget 7 49435-49449. (https://doi.org/10.18632/ oncotarget.10356)

Scheller EL, Troiano N, Vanhoutan JN, Bouxsein MA, Fretz JA, Xi Y, Nelson T, Katz G, Berry R, Church CD, et al. 2014 Use of osmium tetroxide staining with microcomputerized tomography to visualize and quantify bone marrow adipose tissue in vivo. Methods in Enzymology 537 123-139. (https://doi.org/10.1016/B978-0-12-411619-1.00007-0)
Simoncini T, Hafezi-Moghadam A, Brazil DP, Ley K, Chin WW \& Liao JK 2000 Interaction of oestrogen receptor with the regulatory subunit of phosphatidylinositol-3-OH kinase. Nature 407 538-541. (https://doi. org/10.1038/35035131)

Storey NM, O'Bryan JP \& Armstrong DL 2002 Rac and Rho mediate opposing hormonal regulation of the ether-a-go-go-related potassium channel. Current Biology 12 27-33. (https://doi.org/10.1016/S09609822(01)00625-X)

Storey NM, Gentile S, Ullah H, Russo A, Muessel M, Erxleben C \& Armstrong DL 2006 Rapid signaling at the plasma membrane by a nuclear receptor for thyroid hormone. PNAS 103 5197-5201. (https:// doi.org/10.1073/pnas.0600089103)

Van Vliet G, Larroque B, Bubuteishvili L, Supernant K \& Léger J \& Association of Française pour le Dépistage et la Prévention des Handicaps de l'Enfant 2003 Sex-specific impact of congenital hypothyroidism due to thyroid dysgenesis on skeletal maturation in term newborns. Journal of Clinical Endocrinology and Metabolism $\mathbf{8 8}$ 2009-2013. (https://doi.org/10.1210/jc.2002-021735)

Verga Falzacappa C, Petrucci E, Patriarca V, Michienzi S, Stigliano A, Brunetti E, Toscano V \& Misiti S 2007 Thyroid hormone receptor TRbeta1 mediates Akt activation by $\mathrm{T} 3$ in pancreatic beta cells. Journal of Molecular Endocrinology 38 221-233. (https://doi.org/10.1677/ jme.1.02166)

Williams GR \& Bassett JHD 2018 Thyroid diseases and bone health. Journal of Endocrinological Investigation 41 99-109. (https://doi. org/10.1007/s40618-017-0753-4)

Xing W, Govoni KE, Donahue LR, Kesavan C, Wergedal J, Long C, Bassett JD, Gogakos A, Wojcicka A, Williams GR, et al. 2012 Genetic evidence that thyroid hormone is indispensable for prepubertal insulin-like growth factor-I expression and bone acquisition in mice. Journal of Bone and Mineral Research 27 1067-1079. (https://doi. org/10.1002/jbmr.1551)

Xing W, Cheng S, Wergedal J \& Mohan S 2014 Epiphyseal chondrocyte secondary ossification centers require thyroid hormone activation of Indian hedgehog and osterix signaling. Journal of Bone and Mineral Research 29 2262-2275. (https://doi.org/10.1002/ jbmr.2256)

Xing W, Aghajanian P, Goodluck H, Kesavan C, Cheng S, Pourteymoor S, Watt H, Alarcon C \& Mohan S 2016 Thyroid hormone receptor- $\beta 1$ signaling is critically involved in regulating secondary ossification via promoting transcription of the Ihh gene in the epiphysis. American Journal of Physiology: Endocrinology and Metabolism 310 E846-E854. (https://doi.org/10.1152/ajpendo.00541.2015)

Received in final form 6 June 2019

Accepted 28 June 2019

Accepted Preprint published online 28 June 2019 https://joe.bioscientifica.com https://doi.org/10.1530/JOE-19-0172 (c) 2019 Society for Endocrinology Published by Bioscientifica Ltd. Printed in Great Britain 